\title{
An observational case series of targeted virtual geriatric medicine and palliative care consults for hospitalized older adults with COVID-19
}

\author{
Jennifer A. Ouellet ${ }^{1}$, Elizabeth H. Prsic ${ }^{2}$, Rebecca A. Spear ${ }^{1,2,3}$, Leslie Blatt ${ }^{2}$, Shannon Kukulka ${ }^{1}$, \\ Rosemary Cronin-Ozyck ${ }^{2}$, Jennifer M. Kapo ${ }^{2}$, James M. Lai ${ }^{1}$
}

${ }^{1}$ Yale School of Medicine, Section of Geriatrics, Department of Internal Medicine, New Haven, CT, USA; ${ }^{2}$ Yale School of Medicine, Palliative Care Program, Department of Internal Medicine, New Haven, CT, USA; ${ }^{3}$ Maine Dartmouth Family Medicine Residency, Augusta, ME, USA

Contributions: (I) Conception and design: All authors; (II) Administrative support: All authors; (III) Provision of study materials or patients: None; (IV) Collection and assembly of data: All authors; (V) Data analysis and interpretation: All authors; (VI) Manuscript writing: All authors; (VII) Final approval of manuscript: All authors.

Correspondence to: Jennifer A. Ouellet. Assistant Professor, Yale University School of Medicine, E.S. Harkness Memorial Hall, Building A, 367 Cedar Street, Room 307A, New Haven, CT 06510, USA. Email: Jennifer.Ouellet@yale.edu.

\begin{abstract}
Background: COVID-19 presents unique challenges to the care of hospitalized older adults, including fractured lines of communication and uncertainty surrounding long term trajectories in cognition and function. Geriatric medicine and palliative care clinicians bring specialized training in facilitating communication in the face of uncertainty. Insurance expansion of virtual visits enabled inpatient virtual consultation, which can preserve personal protective equipment and minimize exposure to clinicians. We examined changes in goals of care and code status following an inpatient virtual consultation with geriatric medicine and palliative care clinicians.
\end{abstract}

Methods: This was an observational case series study performed at a large tertiary Academic Hospital. The study population included 78 patients aged 65 years and older, hospitalized with COVID-19 who had an inpatient consultation completed by geriatric medicine or palliative care clinicians between April 9, 2020 through May 9, 2020. The intervention was targeted, virtual geriatric medicine or palliative care consultation. All patients admitted to a medical floor with COVID-19 were screened four days a week and if a patient was over the age of 65 , the medical team was offered a consultation by geriatric medicine (ages 80 and above) or palliative care (ages 65-79). Consultation included medical record review, telephone conversations with clinicians and nurses, telephone or video conversations with patients and/or surrogate decision-makers and collaborative case review on daily virtual huddles with an interprofessional team of geriatric medicine and palliative care clinicians. Descriptive statistics were applied to categorize outcomes after chart abstraction.

Results: Following consultation, 24 patients (31\%) patients changed their code status to less invasive interventions. Of patients who were FULL CODE at the time of consultation $(n=42), 2(4.8 \%)$ transitioned to DNR only and $16(38.1 \%)$ transitioned to DNR/DNI after consultation. While 8 patients $(10.3 \%)$ utilized intensive care unit (ICU) level of care prior to consultation, 6 (7.6\%) patients utilized ICU after consultation. After consultation, 11 (14.1\%) patients were referred to hospice.

Conclusions: Given uncertain trajectories in older adults hospitalized with COVID-19 and variability in patient preferences, virtual goals of care geriatric medicine and palliative care consultations should be considered as a key component of COVID-19 hospital protocols.

Keywords: Geriatrics; palliative care; hospital communication; patient centered care

Submitted Jan 15, 2021. Accepted for publication May 13, 2021.

doi: $10.21037 /$ apm-21-117

View this article at: http://dx.doi.org/10.21037/apm-21-117 


\section{Introduction}

Goal-concordant care, supported by patients, caregivers and the National Academy of Medicine (1), includes aligning healthcare interventions with individual patient health outcome goals and healthcare preferences. Goal concordant care is often nuanced, as patients, including older adults vary in the outcomes that matter most to them (2), and the interventions they will accept to achieve them. The COVID-19 pandemic presents clinicians with additional challenges to providing goal concurrent care, including uncertainty regarding expected long-term cognitive and functional trajectories after recovery, fractured lines of communication due to isolation protocols and visitor restrictions, and potential impaired patient capacity for decision making. Further, hospitals have increasingly relied on clinicians with varied levels of training in inpatient internal medicine to cope with surges of hospitalized patients. Such clinicians likely have varied levels of training, experience and comfort with prognostication and discussing complex goals of care, and as such, may benefit from additional support. These outlined challenges may limit the ability of inpatient teams to carry out the critically important goals of care discussions necessary to ensure that patients hospitalized with COVID-19 receive care consistent with their goals and preferences $(3,4)$.

Geriatric medicine and palliative care consultants bring specialized training in facilitating discussions in medicallycomplex older adults with serious illness and uncertainty. To maximize access to specialist care and efficiency of visits, and given concerns surrounding personal protective equipment stores, many health systems utilized the expansion in insurance coverage of virtual visits during the COVID-19 pandemic $(5,6)$.

To support inpatient medical floor teams, patients and surrogate decision makers during the first wave of the COVID-19 pandemic, we created a virtual, targeted consultation service for hospitalized non-intensive care unit (ICU) patients. Prior studies investigating outcomes of targeted palliative care consultations in the ICU (7) and emergency department (8) found a significant transition to less aggressive code status, increased utilization of hospice care, and decreased ICU utilization. Given the challenges to inpatient medical teams outlined above, we focused on older adults admitted to medical floors. This observational case series study describes the characteristics and outcomes of the patients who received targeted, virtual geriatric medicine or palliative care consultation at a large tertiary academic hospital. We present the following article in accordance with the STROBE reporting checklist (available at http://dx.doi.org/10.21037/apm-21-117).

\section{Methods}

\section{Needs assessment and intervention development}

We conducted key informant interviews with clinicians, educators and hospital staff to assess perceived needs; this included geriatric medicine and palliative care clinicians and educators, hospitalist physicians, advanced practice provider clinicians, and medical ICU clinicians. Based on these interviews, a need for additional support to inpatient medical teams in clarifying and defining the goals of care in high risk, non-critically ill, hospitalized older adults was identified. To directly address concerns regarding preservation of health system personal protective equipment and the efforts to minimize clinician workforce COVID-19 exposure, we employed a virtual platform for conducting goals of care consultations as our primary approach as deemed appropriate. Consequently, we specifically developed a virtual, targeted goals of care consultation model for older adults hospitalized with COVID-19 on non ICU-medical floors. A conversation guide was developed based on existing scripts from VitalTalk ${ }^{\odot}(9)$, Prepare for Your Care ${ }^{\odot}$ (10), Respecting Choices ${ }^{\odot}$ (11) and the Conversation Project (12). Two, one-hour virtual training sessions were held with geriatric medicine and palliative care clinicians to practice using these resources to guide discussions.

\section{Study sample}

All hospitalized patients at Yale New Haven Hospital were screened 4 days per week from April 9, 2020 to May 9, 2020 for inclusion criteria: COVID-19 positive patients $\geq 65$ years old on non-ICU medical floors. If the primary team assented to consultation, consultants reviewed the patient, and excluded those without acute goals of care needs (i.e., patient was comfort measures only or clinically stable and discharge planned in the subsequent $24 \mathrm{hrs}$ ). Patients with COVID-19 disease severity requiring medical ICU or medical step down unit level of care were excluded from this study.

\section{Intervention}

Geriatric medicine clinicians provided consultation 
for patients $\geq 80$ years old and palliative care clinicians completed consultation for patients ages 65-79. Consultation included medical record review, telephone conversations with clinicians and nurses, telephone or video conversations with patients and/or surrogate decision-makers and collaborative case review with an interprofessional team of geriatric medicine and palliative care clinicians (attending physicians and fellows, APRNs, social workers, chaplains, and a pharmacist). In performing video consultations with patients, we utilized several electronic platforms including zoom (over IPad, assisted by bedside nurse), a hospital based virtual visit system [InTouch Health (13)] and FaceTime via IPad or IPhone. InTouch Health technology included bedside monitors residing at the patient bedside that could be used throughout the day by any clinician providing care to the patient. Telephone conversations were the first line of contact to patient surrogate decision makers, however we also offered zoom or FaceTime if preferred.

Through the use of virtual platforms, the inpatient consultation teams were able to increase baseline staffing during the COVID-19 pandemic. The geriatric medicine consultation service, typically staffed by 1-2 full time MDs and 1 fellow, was staffed by 2-3 full time MDs, 1 APRN and 1-3 fellows during the implementation of this intervention. The palliative care consultation service, had no changes to the baseline staffing of MD, APRN, SW and chaplain clinicians. Notably, the volume of traditional consultations was less due to the hospital census being proportionately higher with patients with COVID-19 infection.

\section{Adjudication of patient characteristics and outcomes}

The study was conducted in accordance with the Declaration of Helsinki (as revised in 2013). The study was approved by The Ethics Board of The Yale School of Medicine (No. 2000028177) and by The Yale School of Medicine COVID-19 Research Committee. Informed consent was waived by the Yale IRB. Geriatric medicine and palliative care clinicians performed chart abstractions using a standardized data collection form in REDCap (Research Electronic Data Capture), an electronic database management system hosted at Yale School of Medicine $(14,15)$. REDCap is a secure, web-based software platform designed to support data capture for research studies, providing (I) an intuitive interface for validated data capture; (II) audit trails for tracking data manipulation and export procedures; (IIII) automated export procedures for seamless data downloads to common statistical packages; and (IV) procedures for data integration and interoperability with external sources.

Clinicians did not abstract charts of patients for which they had direct contact with the patient or surrogate decision maker. The primary outcome was change in code status following consultation. Chart abstraction was completed for patients by June 9 and thus the outcomes were reported as of that date, for a total follow-up period of 30 days.

Patient comorbidities were determined through review of electronic medical record (EMR) notes and problem lists. Baseline functional status (i.e., IADL, ADL impairments) was determined by EMR review (problem list, review of case management and physical therapy notes, review of clinician notes) and through discussion with surrogate decision makers. Diagnosis of baseline probable dementia was determined by EMR problem list or discussion with surrogate decision maker (confirmation of presence of cognitive concerns and functional impairments due to cognition). Presence of in-hospital delirium was ascertained through review of progress and nursing notes for elements of the Confusion Assessment Method (CAM) including: (I) acute change in and fluctuations in mental status, and (II) inattention and either (III) disorganized thinking or (IV) altered level of consciousness $(16,17)$. Decision making capacity was determined by conversation with the primary medical team and attempted conversation with patients to assess their mental status and ability to understand medical information.

\section{Statistical analysis}

Descriptive statistics determined the frequencies of baseline characteristics and outcomes. We did not require subgroup analysis to meet our study objectives.

\section{Results}

Of the 120 patients who met inclusion criteria, the primary team assented to consultation for 96. After clinician review, 78 patients received consultation (Consort Diagram). No patients were lost to follow-up and there were no elements of missing data. The mean age was 81 and more than two thirds (69.2\%) were White/Caucasian. Approximately half $(52.5 \%)$ were female, and the vast majority (91\%) had more than 2 chronic medical conditions (Table 1). Comorbidities included hypertension (83.3\%), probable dementia (50\%), 
Table 1 Baseline characteristics $(\mathrm{N}=78)$

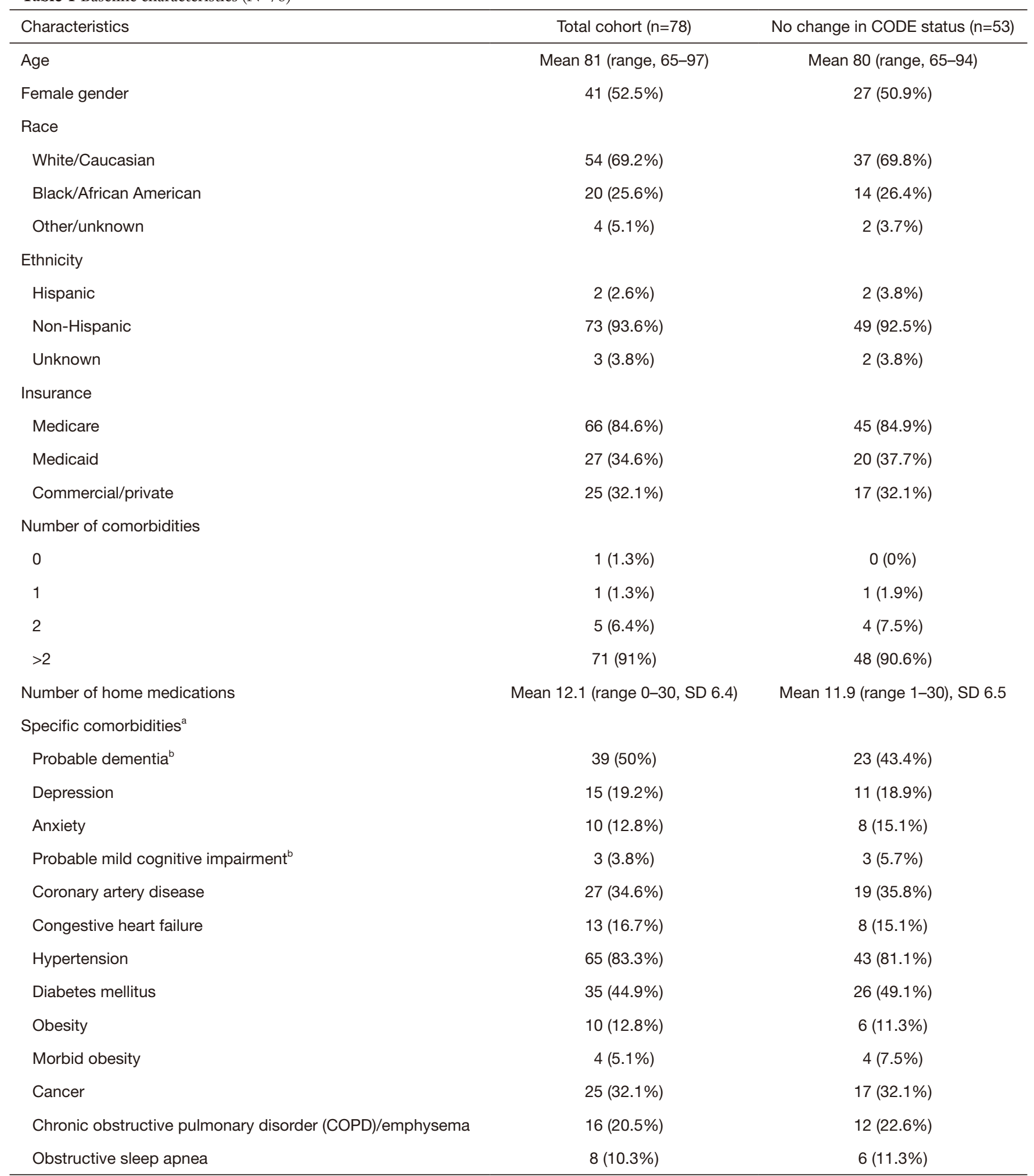

Table 1 (continued) 
Table 1 (continued)

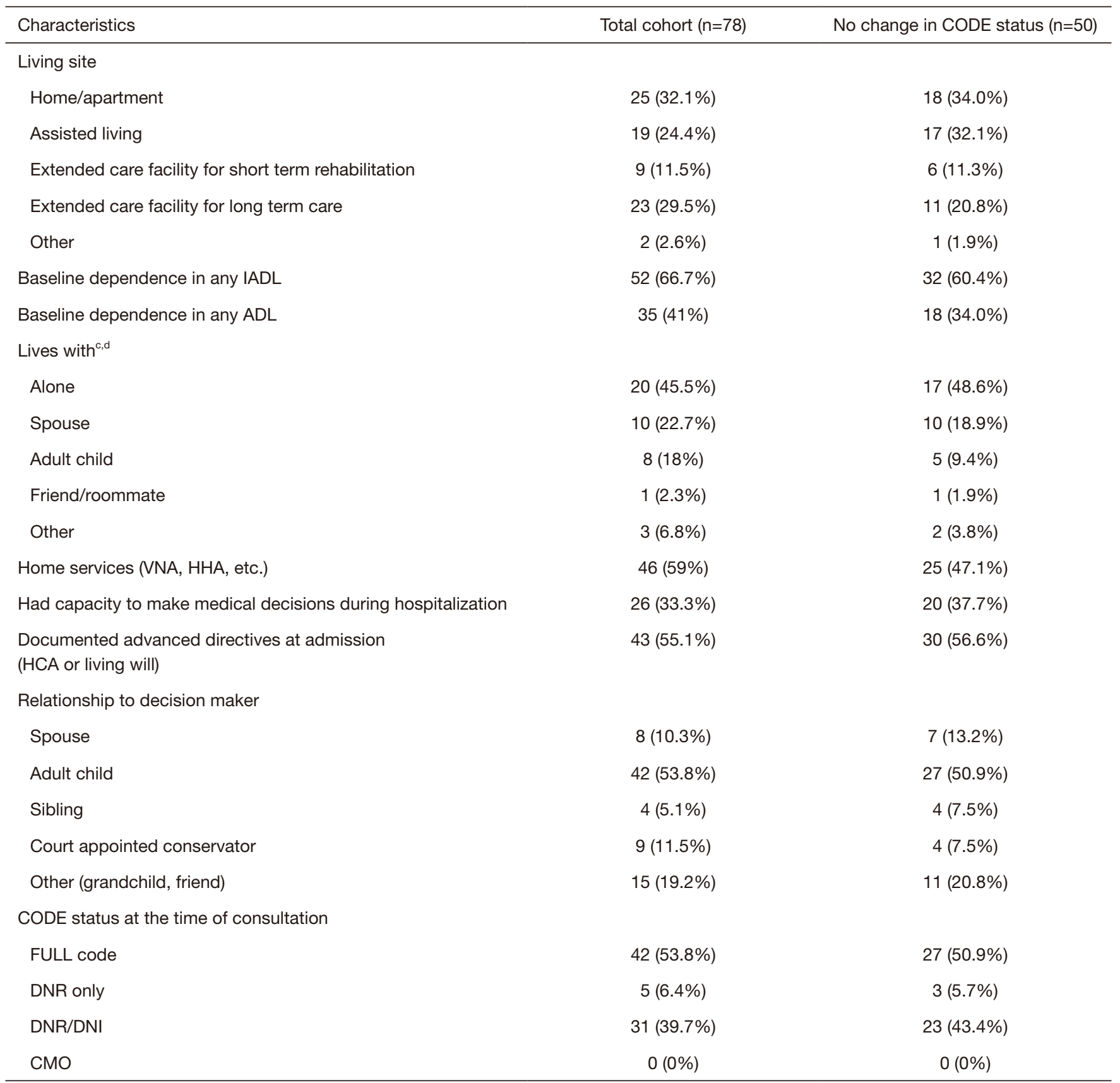

${ }^{a}$ Comorbidities identified in electronic medical record problem list and clinician notes. ${ }^{b}$ Diagnosis either through chart review or discussion with surrogate decision maker. ${ }^{c}$ Numbers calculated out of total living in assisted living or home, $n=42$ for total cohort and $n=35$ for no change in code status. ${ }^{d}$ For total cohort, data missing for 2 patients living in home/apartment or assisted living facility.

diabetes mellitus (44.9\%), coronary artery disease (34.6\%), cancer $(32.1 \%)$ and chronic obstructive pulmonary disorder $(20.5 \%)$. Residence prior to hospitalization included private home/apartment (32.1\%), assisted living (24.4\%), extended care facility (ECF) for long term care $(29.5 \%)$ and
ECF for short term rehabilitation (11.5\%). Notably, one third $(33.3 \%)$ of patients met clinical criteria for delirium, and two-thirds $(66.7 \%)$ of the sample required surrogate decision makers to make medical decisions due to lack of patient capacity. 
Comparison of the demographic characteristics of patients who did not have a change in code status following consultation are compared to the demographic characteristics of the entire cohort in Table 1. There were no substantial differences in age, gender, insurance status, number of comorbidities or mean number of medications. There was a slightly lower proportion of patients with probable dementia in the subset of patients without a code status change. Additionally, there were a higher number of patients residing in assisted living and fewer residents of nursing facilities within the subset of patients who did not change code status. There were lower proportions of patients with dependence in ADLs and IADLs in the subset of patients without code status change compared to the entire cohort.

Mean length of stay of hospitalization was 16.3 days and $13(16.7 \%)$ patients died during hospitalization (Table 2). The median hospital day of geriatric medicine or palliative care consultation was 7.5. Patients had a median of 2.5 virtual consultant visits. Following consultation, 24 patients (31\%) patients changed their code status to less invasive interventions. Of patients who were FULL CODE at the time of consultation $(\mathrm{n}=42), 2(4.8 \%)$ transitioned to DNR only and 16 (38.1\%) transitioned to DNR/DNI after consultation. Eleven patients $(14.1 \%)$ were referred to hospice after consultation. Prior to consultation, 8 (10.3\%) patients required ICU resources, while only 6 (7.6\%) patients used ICU resources after consultation.

\section{Discussion}

We found that almost one third of all older adults hospitalized on a medical floor with COVID-19 opted for a code status change to less invasive measures after virtual consultation with geriatric medicine or palliative care clinicians. This is significant, as despite high mortality in mechanically-ventilated older adults with COVID-19 (18) and uncertain long-term trajectories, there is substantial variability in patient and surrogate decision-maker willingness to decline potentially life-sustaining interventions. Defaulting to the most invasive measures, however, may cause undue suffering for patients who prefer comfort-focused care in the face of poor odds of recovery.

Exploring the willingness to pursue invasive interventions in the context of an uncertain prognosis is one critical component in addressing the overall goals of care for older adults hospitalized with COVID-19. Studies have previously demonstrated that even when older adults have considered their wishes in serious illness and at the end of life, documentation of wishes and communication with surrogate decision maker and health professionals is inadequate (19). Due to increased demands and fractured communication lines, inpatient medical teams may not be able to carry out the critical goals of care conversations necessary to ensure that care older adults receive is consistent with their overall goals and wishes. We provided a valuable resource to patients, surrogate decision makers and primary medical teams through our targeted, virtual consultation model.

The main limitations of our study include use of a single site and its observational design. Our acute efforts to provide support to all hospitalized older adults, their caregivers and the medical teams caring for them, limited our ability to consider randomized designs. While our study population had similarities with previously published cohorts of older adults hospitalized with COVID-19, the majority of our study sample were White/Caucasian race. Although our intervention used age as the primary inclusion criteria due to the unique needs of the older adult population, it is critical to acknowledge that many other factors, notably race, also infer higher risk for disparities in health equity (20). These factors and potential interventions will need to be considered further in future research. We also observed a high rate of probable dementia. This was potentially attributable to geriatric medicine and palliative care consultants uncovering a previously unknown diagnosis of probable dementia or mild cognitive impairment in conversation with surrogate decision makers.

There was a decrease in the proportion of patients who were FULL CODE between the time of admission and the time of initial geriatric medicine or palliative care consultation (Table 2). This change was likely the result of primary team communication with patient and surrogate decision makers regarding overall goals of care. Despite primary team communication regarding goals of care, we observed additional patients transitioning to goals of care directed at less invasive interventions following consultation, in concordance with their values and treatment preferences. We do, therefore, hypothesize an association between geriatric medicine and palliative care consultation and improved goal concordant care given the marked change in patient/surrogate code status decisions made after a median of 7.5 days post admission. We attribute this association with consultant communication anchoring in individual patient goals and care preferences in the context of the current clinical status and likely clinical trajectory. 
Table 2 Hospital course and patient outcomes (N=78)

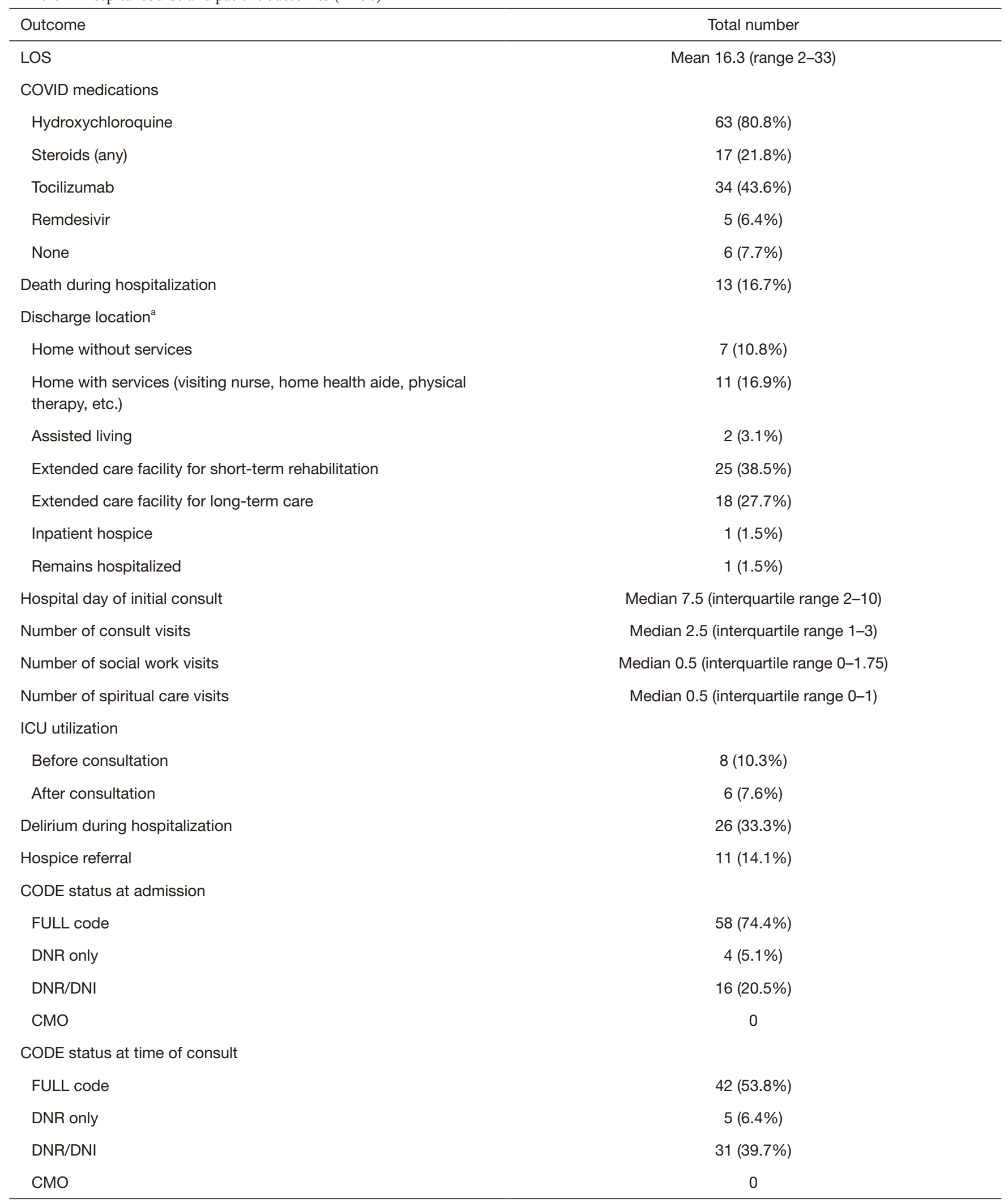

Table 2 (continued) 
Table 2 (continued)

\begin{tabular}{lc}
\hline Outcome & Total number \\
\hline Change in Goals of Care and CODE status after consult stratified by CODE status at the time of consult \\
FULL CODE at time of consult & $2(4.8 \%)$ \\
Change to DNR only & $16(38.1 \%)$ \\
Change to DNR/DNI & $24(57.1 \%)$ \\
No change & 5 \\
DNR at the time of consult & $1(20 \%)$ \\
Change to DNR/DNI & $1(20 \%)$ \\
Change to CMO & $3(60 \%)$ \\
No change & 31 \\
DNR/DNI at the time of consult ${ }^{\mathrm{b}}$ & $4(12.9 \%)$ \\
Change to CMO & $1(3.2 \%)$ \\
Change to FULL CODE & $26(83.9 \%)$ \\
No change & $3(2 \%)$
\end{tabular}

${ }^{a}$ Total percentage calculated out of the 65 patients who were discharged alive from the hospital. ${ }^{b}$ Of those who were DNR/DNI at the time of consult, 3 patients did not change code status formally but opted to not escalate care in the event of decompensation.

Though, we are not powered to detect statistical significance between the demographic characteristics between subsets of patients in our cohort (i.e., patients who changed their code status $v s$. those patients who did not), we did find several notable observations. Those patients who did not change their code status appeared to be less likely to live in nursing home settings and have less ADL dependencies. This likely reflects that more functional patients were more willing to pursue invasive interventions, possibly due to perceptions of likely recovery from acute illness.

Through expansion of technology enabling video visits and expansion of insurance coverage for such visits, we were able to use virtual platforms for inpatient visits during the COVID-19 pandemic. The benefits of this model include expansion of access, preservation of personal protective equipment, and reduced potential workforce exposure. While many patients were able to engage successfully with video visits, there were some barriers in terms of hearing impairment and patient understanding due to cognitive impairment. On balance, there were some increased demands placed on bedside nurses and staff to assist with the technology; however, we coordinated consultations to occur during usual nursing care encounters to minimize this burden.

Targeted consults using a virtual platform to address goals of care in non-ICU, hospitalized older adults with
COVID-19 proved feasible and provided support to primary medical teams, the patients, and their surrogate decision makers during a particularly high stress period. This intervention was particularly valuable for clinicians without formal communication skill training or those who do not routinely practice hospital based medicine. The virtual platform of this consult model increased access to geriatric and palliative care resources while limiting overall exposure risk to clinicians and conserving hospital personal protective equipment. Given changes to billing codes and expansion of supportive infrastructure, this model may be applied to hospital protocols even after the COVID-19 pandemic to improve access to specialty care.

COVID-19 cases and hospitalizations have persisted throughout the United States $(21,22)$ which continues to place strain on hospital systems and clinicians. Our study demonstrates that we were able to successfully support primary medical teams, patients, and surrogate decision makers, with access to consultant care through a virtual platform designed to promote goal concordant care and overcome staffing and communication barriers created by restrictions associated with the COVID-19 pandemic. Based on these findings, virtual geriatric medicine and palliative care consultations should be considered as a key component of COVID-19 and pandemic hospital protocols. 


\section{Acknowledgments}

The authors would like to acknowledge the hard work of the inpatient geriatric medicine and palliative care teams at Yale New Haven Hospital for contributing to the care of older adults hospitalized with COVID-19. In addition to the authors of this study, team members include: Alessandra Capobianco, MD; Geraldine Fabregas, MD; Laura Moreines, APRN; Mary E. Tinetti, MD; Leo Cooney, MD; Richard Marottoli, MD; Gregory Ouellet, MD; Loni R. Belyea, MD; Jennifer Freimund, MD; Zehra Omer, MD; Chisom Ikeji, MD; Gena Lennon Gomez, LCSW; Ed Schwartz, LCSW; Christina Holt, APRN; Kelly Cronin, APRN; Julie Palmer Hoffman, APRN; Andrew Putnam, MD; Mark Swidler, MD; Dmitry Kozhevnikov, DO; Laura Morrison, MD; Alex Mbewe, MD; Chris Ahern, DO; Leah Tenenbaum, Chaplain; Trinh Bui, PharmD; Kelly Ford, LCSW; Andrea Lucibello, LCSW.

Funding: HRSA Yale GWEP 6 U1QHP28745-03-06.

\section{Footnote}

Reporting Checklist: The authors have completed the STROBE reporting checklist. Available at http://dx.doi. org/10.21037/apm-21-117

Data Sharing Statement: Available at http://dx.doi. org/10.21037/apm-21-117

Peer Review File: Available at http://dx.doi.org/10.21037/ apm-21-117

Conflicts of Interest: All authors have completed the ICMJE uniform disclosure form (available at http://dx.doi. org/10.21037/apm-21-117). The authors have no conflicts of interest to declare.

Ethical Statement: The authors are accountable for all aspects of the work in ensuring that questions related to the accuracy or integrity of any part of the work are appropriately investigated and resolved. The study was conducted in accordance with the Declaration of Helsinki (as revised in 2013). The study was approved by Ethics Board of Yale School of Medicine (No. 2000028177) and by the Yale School of Medicine COVID-19 Research Committee. Informed consent was waived by the Yale IRB.

Open Access Statement: This is an Open Access article distributed in accordance with the Creative Commons Attribution-NonCommercial-NoDerivs 4.0 International License (CC BY-NC-ND 4.0), which permits the noncommercial replication and distribution of the article with the strict proviso that no changes or edits are made and the original work is properly cited (including links to both the formal publication through the relevant DOI and the license). See: https://creativecommons.org/licenses/by-nc-nd/4.0/.

\section{References}

1. Dzau VJ, McClellan MB, McGinnis JM, et al. Vital Directions for Health and Health Care: Priorities From a National Academy of Medicine Initiative. JAMA 2017;317:1461-70.

2. Fried TR, Tinetti ME, Iannone L, et al. Health Outcome Prioritization as a Tool for Decision Making among Older Persons with Multiple Chronic Conditions. Arch Intern Med 2011;171:1854-6.

3. Curtis JR, Kross EK, Stapleton RD. The Importance of Addressing Advance Care Planning and Decisions About Do-Not-Resuscitate Orders During Novel Coronavirus 2019 (COVID-19). JAMA 2020;323:1771-2.

4. Prsic EH, Ouellet JA, Lai JM. The value of triggered geriatrics and palliative care consults in hospice medicine during the COVID-19 response. Ann Palliat Med 2021;10:970-2.

5. Ankuda CK, Woodrell CD, Meier DE, et al. A Beacon For Dark Times: Palliative Care Support During the Coronavirus Pandemic. NEJM Catalyst 2020. Available online: https://catalyst.nejm.org/doi/full/10.1056/ CAT.20.0204

6. Nakagawa S, Berlin A, Widera E, et al. Pandemic Palliative Care Consultations Spanning State and Institutional Borders. J Am Geriatr Soc 2020;68:1683-5.

7. Zalenski RJ, Jones SS, Courage C, et al. Impact of Palliative Care Screening and Consultation in the ICU: A Multihospital Quality Improvement Project. J Pain Symptom Manage 2017:53:5-12.e13.

8. Lee J, Abrukin L, Flores S, et al. Early Intervention of Palliative Care in the Emergency Department During the COVID-19 Pandemic. JAMA Intern Med 2020;180:1252-4.

9. Vital Talk [Internet]. Available online: https://www. vitaltalk.org/

10. Prepare for Your Care [Internet]. Available online: https:// prepareforyourcare.org/welcome.

11. Respecting Choices [Internet]. Available online: https:// 
respectingchoices.org/.

12. The Conversation Project COVID-19 Resources [Internet]. Available online: https://theconversationproject. org/covid19/

13. In Touch Health [Internet]. Available online: https:// intouchhealth.com/

14. Harris PA, Taylor R, Thielke R, et al. Research electronic data capture (REDCap)--a metadata-driven methodology and workflow process for providing translational research informatics support. J Biomed Inform 2009;42:377-81.

15. Harris PA, Taylor R, Minor BL, et al. The REDCap consortium: Building an international community of software platform partners. J Biomed Inform 2019;95:103208.

16. Inouye SK, van Dyck CH, Alessi CA, et al. Clarifying confusion: the confusion assessment method. A new method for detection of delirium. Ann Intern Med 1990;113:941-8.

17. Inouye SK, Leo-Summers L, Zhang Y, et al. A chart-based method for identification of delirium: validation compared with interviewer ratings using the confusion assessment method. J Am Geriatr Soc 2005;53:312-8.

18. Richardson S, Hirsch JS, Narasimhan M, et al. Presenting Characteristics, Comorbidities, and Outcomes Among 5700 Patients Hospitalized With COVID-19 in the New York City Area. JAMA 2020;323:2052-9.

19. Heyland DK, Barwich D, Pichora D, et al. Failure to engage hospitalized elderly patients and their families in advance care planning. JAMA Intern Med 2013;173:778-87.

20. Bassett MT, Chen JT, Krieger N. Variation in racial/ ethnic disparities in COVID-19 mortality by age in the United States: A cross-sectional study. PLoS Med 2020;17:e1003402.

21. CDC COVID-19 Data Tracker. [Internet]. Available online: https://covid.cdc.gov/covid-data-tracker/

22. Johns Hopkins Coronavirus Resource Center [Internet]. Available online: https://coronavirus.jhu.edu/map.html

Cite this article as: Ouellet JA, Prsic EH, Spear RA, Blatt L, Kukulka S, Cronin-Ozyck R, Kapo JM, Lai JM. An observational case series of targeted virtual geriatric medicine and palliative care consults for hospitalized older adults with COVID-19. Ann Palliat Med 2021;10(6):6297-6306. doi: 10.21037/ apm-21-117 